\title{
Ti:sapphire-pumped deep-infrared femtosecond optical parametric oscillator based on $\mathrm{CdSiP}_{2}$
}

\author{
V. Ramaiah-Badarla, ${ }^{1}$ S. Chaitanya Kumar, ${ }^{1}$ A. Esteban-Martin, ${ }^{1}$ \\ K. Devi, ${ }^{1}$ K. T. ZaWilski, ${ }^{2}$ P. G. Schunemann, ${ }^{2}$ M. Ebrahim-Zadeh ${ }^{1,3,}{ }^{*}$ \\ ${ }^{1}$ ICFO-Institut de Ciencies Fotoniques, The Barcelona Institute of Science and Technology, 08860 Castelldefels (Barcelona), Spain \\ ${ }^{2}$ BAE Systems, Incorporated, MER15-1813, P.O. Box 868, Nashua, New Hampshire 03061-0868, USA \\ ${ }^{3}$ Institucio Catalana de Recerca i Estudis Avancats (ICREA), Passeig Lluis Companys 23, Barcelona 08010, Spain \\ *Corresponding author: majid.ebrahim@icfo.es
}

Received XX Month XXXX; revised XX Month, XXXX; accepted XX Month XXXX; posted XX Month XXXX (Doc. ID XXXXX); published XX Month XXXX

\begin{abstract}
We report a femtosecond optical parametric oscillator (OPO) for the deep-infrared (deep-IR) based on the Kerrlens-mode-locked (KLM) Ti:sapphire laser as the pump source. By deploying a novel cascaded intracavity arrangement comprising a femtosecond OPO based on the nonlinear crystal, $\mathrm{CdSiP}_{2}$ (CSP), synchronously pumped internal to a Mg0:PPLN femtosecond OPO, we have generated broadly tunable radiation across 5958$8117 \mathrm{~nm}$ using rapid static cavity delay tuning, with a maximum power of $64 \mu \mathrm{W}$ at $6791 \mathrm{~nm}$, limited by the absorption in mirror substrates as well as polarizationdependent intracavity losses. The deep-IR idler power exhibits excellent passive stability of better than $1.1 \%$ rms over 2 hours, with a spectral bandwidth as large as $\sim 650 \mathrm{~nm}$ at $\sim 6800 \mathrm{~nm}$. The demonstrated concept is generic and can be similarly deployed in other operating time-scales and wavelength regions, also using different laser pump sources and nonlinear materials. (c) 2015 Optical Society of America
\end{abstract}

OCIS codes: (190.4360) Nonlinear optics, devices; (190.7110) Ultrafast nonlinear optics; (190.4400) Nonlinear optics, materials; (190.4970)

Parametric oscillators and amplifiers.

http://dx.doi.org/10.1364/OL.99.099999

Coherent ultrafast laser sources in the mid-infrared (mid-IR) are of interest for a wide range of applications including spectroscopy and trace gas sensing [1,2]. With the scarcity of ultrafast solid-state lasers, synchronously-pumped optical parametric oscillators (OPOs) represent a highly effective approach to the generation of ultrashort pulses in this spectral region [3]. Using the Kerr-lens-mode-locked (KLM) Ti:sapphire laser as the pump source, femtosecond OPOs in various configurations, covering spectral regions from below $\sim 1 \mu \mathrm{m}$ to beyond $\sim 4 \mu \mathrm{m}$, have been extensively developed by exploiting oxidebased birefringent nonlinear crystals such as $\mathrm{KTiOPO}_{4}$, $\mathrm{KTiOAsO}_{4}$, $\mathrm{RbTiOAsO}_{4}$, CsTiOAsO ${ }_{4}$, and $\mathrm{BiB}_{3} \mathrm{O}_{6}[4,5]$, as well as quasi-phasematched (QPM) materials including periodically-poled $\mathrm{LiNbO}_{3}(\mathrm{PPLN})$
[6]. With the advances in fiber laser technology, femtosecond OPOs pumped by ultrafast $\mathrm{Yb}$-fiber lasers at $\sim 1060 \mathrm{~nm}$, covering spectral regions from $\sim 1.4 \mu \mathrm{m}$ to above $\sim 4 \mu \mathrm{m}$, have also been successfully realized, addressing new application areas, for example in frequency comb generation [7]. On the other hand, after more than two decades of research and development, the extension of spectral range of femtosecond OPOs significantly beyond $\sim 4 \mu \mathrm{m}$ remains challenging, due to multi-phonon absorption in oxide-based nonlinear crystals. As an alternative, non-oxide QPM crystals such as orientation-patterned GaAs (OP-GaAs) can be potentially exploited for wavelength generation beyond $\sim 4 \mu \mathrm{m}$, but this requires pumping above $\sim 2 \mu \mathrm{m}$ to avoid two-photon absorption (TPA) in this material. Chalcopyrite crystals such as $\mathrm{AgGaSe}$, $\mathrm{AgGaS}_{2}$, and $\mathrm{ZnGeP}_{2}$, can generate deep midIR radiation up to $\sim 10 \mu \mathrm{m}$, but must be pumped well above $\sim 1 \mu \mathrm{m}$ to similarly avoid two-photon and residual absorption, and material quality still remains a major practical issue yet to be overcome.

The newly discovered chalcopyrite nonlinear crystal, cadmium silicon phosphide, $\mathrm{CdSiP}_{2}$ (CSP), has drawn special attention in recent years due to its unique linear and nonlinear optical properties [8]. It is an optically uniaxial crystal with high nonlinear coefficient $\left(d_{\text {eff }} \sim 84 \mathrm{pm} / \mathrm{V}\right)$ and wide transparency ( $\sim 600 \mathrm{~nm}$ to $\sim 6.5 \mu \mathrm{m})$, which allows pumping near $1 \mu \mathrm{m}$ without the onset of TPA. Importantly, CSP has the unique capability for parametric generation beyond $\sim 6 \mu \mathrm{m}$ with direct pumping at $\sim 1 \mu \mathrm{m}$ under type I $(e \rightarrow o o)$ noncritical phase-matching, (NCPM). By exploiting this scheme in CSP, a number of ultrafast picosecond and femtosecond parametric sources have already been successfully demonstrated using mode-locked solid-state and fiber pump lasers at $\sim 1 \mu \mathrm{m}$, providing spectral coverage in the $\sim 6.1-6.7 \mu \mathrm{m}$ range [9-14]. These include a single-pass picosecond parametric generator (OPG) at $\sim 6.2 \mu \mathrm{m}$ pumped by a mode-locked amplified diode-pumped Nd:YVO4 laser at $1064 \mathrm{~nm}$ at $100 \mathrm{kHz}$ repetition rate [9], and an OPG tunable over 6.1-6.7 $\mu$ m pumped by a mode-locked cavity-dumped Nd:YAG laser at $1064 \mathrm{~nm}$ with a pulse repetition rate of $5 \mathrm{~Hz}$ [10]. A picosecond OPO synchronously pumped by a mode-locked amplified Nd:YAG laser at $1064 \mathrm{~nm}$ was also reported, generating idler pulses at $\sim 6.4 \mu \mathrm{m}$ at $100 \mathrm{MHz}$ [11], while a compact high-energy picosecond OPO using a Nd:YAG oscillator-amplifier system at 1064 $\mathrm{nm}$ was demonstrated, providing tunable output across $\sim 6.1-6.6 \mu \mathrm{m}$ at $450 \mathrm{MHz}$ repetition rate [12]. In the femtosecond time-scale, operation 
of an OPO based on CSP synchronously pumped by 130-fs pulses at $1053 \mathrm{~nm}$ from a mode-locked Yb:KYW/Yb:fiber oscillator-amplifier was reported with idler output centered at $\sim 6.2 \mu \mathrm{m}$ at $100 \mathrm{MHz}$ [13]. More recently, a high-power femtosecond OPO synchronously pumped by 560-fs pulses from a Yb:KGW thin-disk solid-state laser system at $1029 \mathrm{~nm}$ was reported with tuning across $6.5-7.2 \mu \mathrm{m}$ at $\sim 43$ $\mathrm{MHz}$ repetition rate [14].

At the same time, the exploitation of the KLM Ti:sapphire laser as the pump source for the development of femtosecond OPOs beyond $\sim 4$ $\mu \mathrm{m}$ offers a number of important merits. The laser, which represents the workhorse of ultrafast technology, still remains the most viable and well-established source of femtosecond pulses, capable of delivering the shortest transform-limited pulse durations and the broadest spectral bandwidths. These features can be of great advantage in also ultimately delivering the shortest pulses and largest spectral bandwidths from OPOs, which are important for applications such as optical frequency comb generation and spectroscopy. Using a 20 -fs KLM Ti:sapphire laser, a synchronously-pumped OPO in singlyresonant oscillator (SRO) configuration was previously demonstrated, providing broadband few-cycle pulses across $\sim 2-4 \mu \mathrm{m}$ in the mid-IR [15]. However, the use of the KLM Ti:sapphire laser in combination with chalcopyrite materials for the development of femtosecond OPOs beyond $\sim 4 \mu \mathrm{m}$ is fundamentally precluded by TPA at pumping wavelengths below $\sim 1 \mu \mathrm{m}$. In an effort to overcome this constraint, external cascaded pumping using two OPOs in series, based on CTA and $\mathrm{AgGaSe}$, was previously deployed, providing tuning up to $\sim 8 \mu \mathrm{m}$ [16]. However, such external tandem schemes inherently require high input pump powers, and result in relatively complex architectures involving two independent OPO cavities synchronized in series to one another, and to the pump laser, which can also lead to output instabilities. As such, alternative strategies need to be devised for the development of femtosecond OPOs for the deep-IR based on the KLM Ti:sapphire_laser as the pump source, but in more simplified and practical designs. Here we report a novel approach to achieve this goal by demonstrating a femtosecond OPO based on the cascaded concept, but deploying an intracavity pumping arrangement, for the first time to our knowledge. In the new scheme, a secondary femtosecond OPO is synchronously pumped internal to a primary OPO, with the two oscillators sharing a common cavity synchronized to a KLM Ti:sapphire pump laser. By deploying MgO:PPLN as the gain material for the primary $\mathrm{OPO}$, we generate signal at a wavelength $>1 \mu \mathrm{m}$, which is then utilized to internally pump a secondary OPO based on CSP, thus avoiding TPA in the crystal. Both OPOs are in SRO configuration, where by taking advantage of the high intracavity signal intensities in the primary OPO, successful operation of the secondary OPO is achieved without the need for a high external pump power. Since the two OPOs also share a common cavity in a composite design, the scheme also results in a simplified system architecture, increased output stability, and rapid static tuning. Although wavelength tuning can be achieved in several ways, such as pump, grating and angle tuning, here we use cavity delay tuning. In this scheme, the CSP femtosecond OPO is rapidly tuned across the entire $\sim 5.9-8.1 \mu \mathrm{m}$ by simple variation of its cavity delay without adjustment of any other parameters. The output exhibits excellent passive power stability better than $1.1 \%$ rms over 2 hours.

The schematic of the experimental setup for the intracavity cascaded femtosecond OPO is shown in Fig. 1. The setup consists of two OPOs sharing a common cavity, in which the signal generated from a primary MgO:PPLN femtosecond OPO acts as the pump for a secondary OPO based on CSP. The MgO:PPLN OPO is synchronously pumped by a KLM Ti:sapphire laser providing $900 \mathrm{~mW}$ of average power in pulses of 155 fs duration at $76 \mathrm{MHz}$ repetition rate. The laser is operated at a central wavelength of $797 \mathrm{~nm}$ and has an output spectrum with a full-width- at-half-maximum (FWHM) spectral bandwidth of $\sim 7 \mathrm{~nm}$. To avoid back-reflections, an isolator is deployed between the laser and the

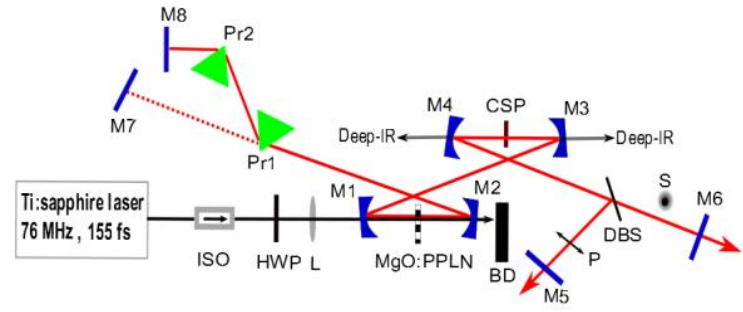

Fig. 1. Experimental setup for the intracavity cascaded OPO. ISO: Faraday isolator, HWP: Half-wave-plate, L: Lens, M1-8: Mirrors, DBS: Dichroic beam-splitter, Pr1-2: Prism pair, BD: Beam dump.

primary $\mathrm{OPO}$ and a half-wave plate provides the required polarization for phase-matching in the MgO:PPLN crystal, which is 0.5-mm-long, 3.4-mm-wide and 1-mm-thick, and maintained at $100{ }^{\circ} \mathrm{C}$ in an oven. The crystal contains a fan-out grating varying in period from $\Lambda=16$ to $23 \mu \mathrm{m}$ across the 3.4-mm-wide aperture, and the end-faces are antireflection $(\mathrm{AR})$-coated $(R<0.75 \%)$ for the signal over $1000-1600$ $\mathrm{nm}$ and pump $(R<5 \%)$ over $720-820 \mathrm{~nm}$, with high transmission $(T>85 \%)$ for the idler over $1600-3500 \mathrm{~nm}$. The secondary OPO is based on a 0.5 -mm-long CSP crystal with a $4 \times 5 \mathrm{~mm}^{2}$ aperture, cut at $\theta=90^{\circ}\left(\varphi=45^{\circ}\right)$ for type I $(e \rightarrow o o)$ NCPM at room temperature. The crystal faces are AR-coated for high transmission over 1020-1300 nm (T>99\%) and 5900-6700 nm (T>95\%). The intracavity cascaded OPO is configured as a standing-wave cavity with two internal foci, where the MgO:PPLN and CSP crystals are located. The resonator is formed by a single set of mirrors comprising four concave ( $r=100 \mathrm{~mm}$ ), M1M4, and four plane, M5-M8, mirrors. All mirrors are made of fused silica substrates and are coated for high transmission (T>90\%) at 710$840 \mathrm{~nm}$ for the KLM Ti:sapphire pump and high reflectivity $(R>99.8 \%)$ over 980-1640 $\mathrm{nm}$ for the signal wavelength range in both MgO:PPLN and CSP, ensuring SRO operation in both OPOs. Using a lens of focal length, $f=7.5 \mathrm{~cm}$, the pump beam is focused to a waist radius of $w_{\mathrm{p}} \sim 25$ $\mu \mathrm{m}$ in the MgO:PPLN crystal, placed at the center of M1-M2. The cavity design results in a signal beam waist of $w_{\mathrm{s} 1} \sim 29 \mu \mathrm{m}$ in both MgO:PPLN as well as the CSP crystal located at the center of M3-M4. The two OPO cavities are separated by a dichroic beam-splitter (DBS) to allow independent control of each resonator length for synchronization with their respective pump pulse trains, since the difference in the refractive index and physical length of the two crystals results in different physical lengths of the cavity for the same round-trip time. The two cavities are then completed by the plane mirrors, M5 and M6, which are mounted on precision translation stages. The DBS is AR-coated for high reflectivity $(R>99 \%)$ over $1000-1100 \mathrm{~nm}$ and high transmission (T>98\%) across $1150-1350 \mathrm{~nm}$, thus reflecting the signal from the MgO:PPLN OPO, while transmitting the signal from the CSP OPO. Since the signal waves generated by the two crystals are orthogonally polarized (as indicated by $S$ and $P$ in Fig. 1), one could ideally also use a polarization-dependent beams-splitter to separate the two cavities. At the common end of the cavity, mirror M7 serves as the end mirror for both the OPOs, with M8 serving as the end mirror in the dispersioncompensated cavity. Dispersion compensation is implemented using a pair of intracavity SF11 prisms to achieve the near-transform-limited signal pulses in the MgO:PPLN OPO to pump the CSP OPO.

Before introducing the CSP crystal into the cavity, we first characterized the signal pulses from the dispersion-compensated MgO:PPLN OPO. Using a 2- $\mu \mathrm{m}$ thin pellicle beam-splitter at Brewster angle located between M4 and DBS, we could extract up to $72 \mathrm{~mW}$ of signal power for measurement of pulse duration. A typical interferometric autocorrelation of the MgO:PPLN signal pulses at 1024 
$\mathrm{nm}$ is shown in Fig. 2. The autocorrelation trace results in a pulse duration of 210 fs (assuming $\operatorname{sech}^{2}$ pulse shape) with a FWHM spectral bandwidth of $7.3 \mathrm{~nm}$, corresponding to a time-bandwidth product, $\Delta \tau \Delta v \sim 0.44$, close to the transform limit.

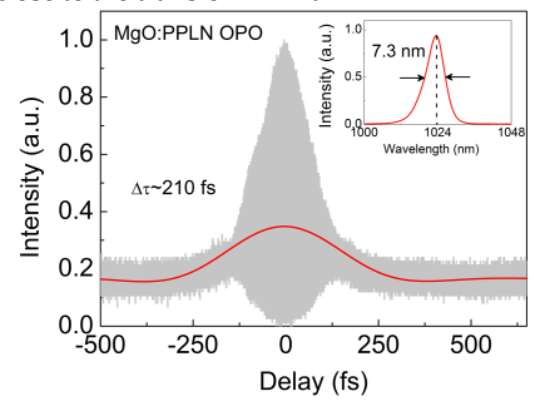

Fig. 2. Interferometric autocorrelation of signal pulses from the MgO:PPLN OPO. Inset: Corresponding spectrum centered at $1024 \mathrm{~nm}$.

After the characterization of the MgO:PPLN OPO signal pulses, we introduced the CSP crystal into the secondary focus of the cavity between M3 and M4. The cavity length of the Mg0:PPLN OPO is adjusted to compensate for the additional path length due to the CSP crystal as well as to achieve the required signal spectral characteristics to pump the CSP OPO. The insertion of the CSP resulted in a minimal drop in the MgO:PPLN signal power from $72 \mathrm{~mW}$ to $68 \mathrm{~mW}$, confirming the low loss and good transmission of the CSP crystal at $1024 \mathrm{~nm}$. The CSP OPO was synchronized to the MgO:PPLN OPO, and hence the pump laser repetition rate, by fine translation of M6, until oscillation was achieved. Under perfect synchronization, the two signal wavelengths from both OPOs could resonate simultaneously inside the composite cavity, as shown in Fig. 3. The signal wavelength generated by the MgO:PPLN OPO is centered at $1024 \mathrm{~nm}$, while that generated by the CSP OPO is centered at $1194 \mathrm{~nm}$. We also observed generation of strong red light from the CSP crystal due to the non-phase-matched second harmonic generation of the signal at $597 \mathrm{~nm}$, as shown in the inset of Fig. 3. The signal pulses generated in the CSP OPO are affected by the dispersion in the Mg0:PPLN crystal, and vice versa. For example, the group delay dispersion experienced by the signal pulses from the CSP OPO at an operating wavelength of $1194 \mathrm{~nm}$ is estimated as $\sim 70$ $\mathrm{fs}^{2}$ considering the ordinary polarization in the MgO:PPLN crystal.

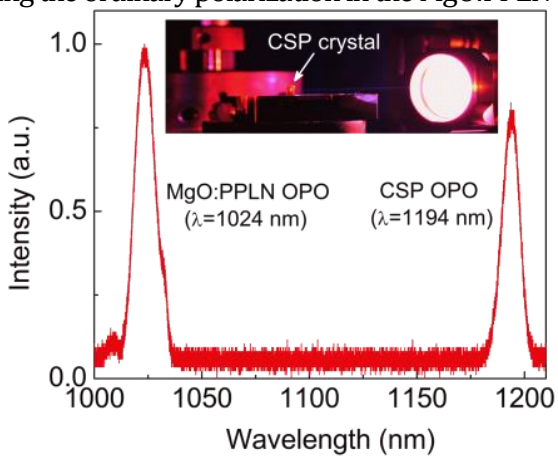

Fig. 3. Simultaneous oscillation of signal wavelengths in MgO:PPLN and CSP OPOs resonant within the composite cavity. Inset: CSP crystal inside the cavity and the bright non-phase-matched red radiation generated by the CSP OPO.

We then systematically characterized the CSP femtosecond OPO. Wavelength tuning in the CSP OPO was achieved only by variation of its cavity length. For a fixed Mg0:PPLN signal wavelength of $1024 \mathrm{~nm}$, we were able to tune the CSP OPO signal across $1172-1237 \mathrm{~nm}$ by changing the cavity delay over $\Delta L \sim 70 \mu \mathrm{m}$ via translation of M6. The corresponding signal spectra are shown in Fig. 4(a). The FWHM signal bandwidth varies from $10.5 \mathrm{~nm}$ at $1172 \mathrm{~nm}$ to $29 \mathrm{~nm}$ at $1237 \mathrm{~nm}$. The corresponding reconstructed idler spectra from the Manley-Rowe relations are shown in Fig. 4(b). As can be seen, the central wavelength of the idler from the CSP OPO can be tuned across 5958-8117 nm (over $2159 \mathrm{~nm}$ ) in the deep-IR, which is to our knowledge the broadest spectral coverage so far achieved with parametric conversion in CSP. Also presented in the inset of Fig. 4(b) is the FWHM idler spectral bandwidth as a function of wavelength, showing a variation from 658 $\mathrm{nm}$ at $5958 \mathrm{~nm}$ to $508 \mathrm{~nm}$ at $8117 \mathrm{~nm}$, with a maximum FWHM bandwidth of $658 \mathrm{~nm}$ centered at $6843 \mathrm{~nm}$.
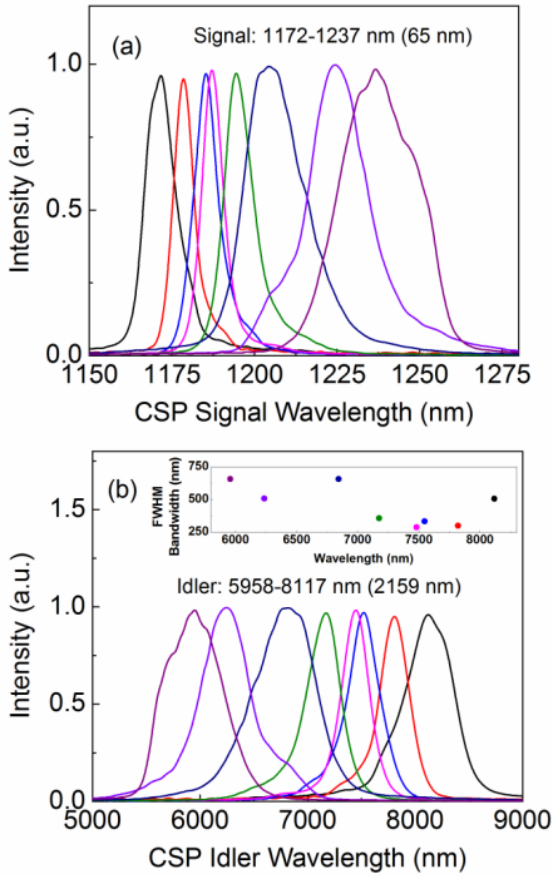

Fig. 4. (a) Signal and (b) reconstructed idler spectra across the CSP OPO tuning range for Mg0:PPLN OPO signal wavelength of $1024 \mathrm{~nm}$.

The signal power leakage through the highly reflecting end mirror, M6, and the corresponding wavelength, measured across the tuning range of the CSP OPO, as a function of the cavity delay, for a MgO:PPLN signal wavelength of $\sim 1022 \mathrm{~nm}$, are shown in Fig. 5. As evident, the signal power varies from $6 \mu \mathrm{W}$ at $1171 \mathrm{~nm}$ for a negative cavity detuning of $\Delta L=-50 \mu \mathrm{m}$ to a maximum of $346 \mu \mathrm{W}$ at $1204 \mathrm{~nm}$ under perfect synchronization, beyond which it drops down to $44 \mu \mathrm{W}$ at $1236 \mathrm{~nm}$ for a positive detuning of $\Delta L=+20 \mu \mathrm{m}$. The corresponding idler wavelengths calculated from energy conservation, vary from 5913 to $8046 \mathrm{~nm}$, as shown in Fig. 5. The data represent the widest tuning together with the longest wavelengths generated from a CSP

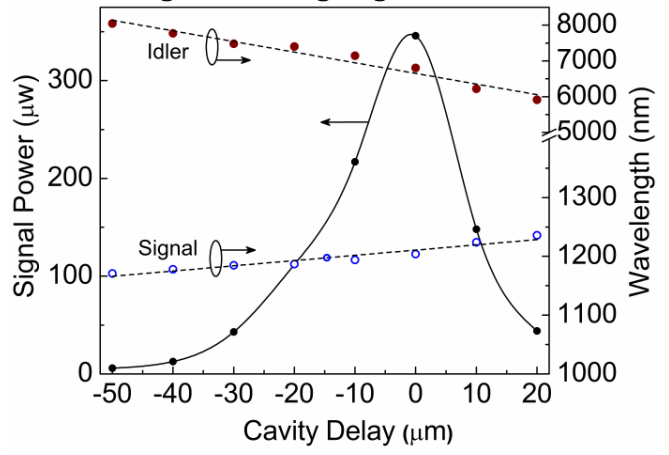

Fig. 5. Signal power and the corresponding CSP OPO signal and idler wavelengths versus the CSP OPO cavity delay. 
OPO in any time scale. The ability to achieve such a performance is one of the main advantages of the intracavity cascaded technique, which enables flexible choice of the pump wavelength. Further, we note that the CSP OPO is operated at room temperature with no temperature control for the CSP crystal.

We also characterized the two OPOs with respect to pump depletion. While pumping at the maximum available Ti:sapphire laser power of $900 \mathrm{~mW}$, we recorded a pump depletion of 36\% in the MgO:PPLN OPO alone, with the CSP OPO detuned well away from synchronization to cease oscillation. However, with both OPOs perfectly synchronized, we recorded an additional pump depletion of $14 \%$, resulting in a total depletion of $50 \%$ in the Ti:sapphire power due to parametric conversion in both OPOs. In order to estimate the change in the intracavity signal power in the MgO:PPLN OPO due to the operation of CSP OPO, we recorded the signal leakage through M5, with and without CSP OPO in oscillation. The result is shown in Fig. 6, where a depletion of $\sim 18 \%$ in the intracavity signal power of MgO:PPLN OPO is evident due to the oscillation of the CSP OPO. Even with this relatively low pump depletion, operation of the CSP OPO was sufficiently robust that we could replace the highly reflecting end mirror, M6, with a 5\% output coupler, where we were able to extract

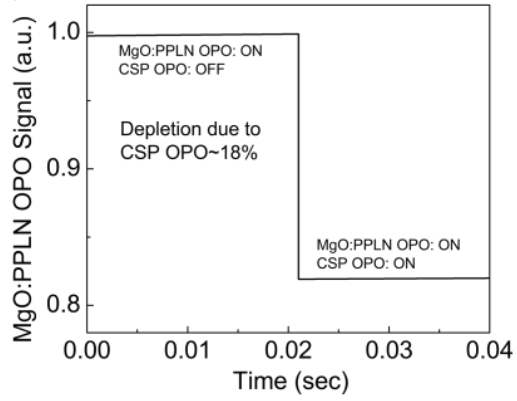

Fig. 6. Depletion of the signal from the MgO:PPLN OPO due the CSP OPO measured using a GaAs photodetector.

up to $9 \mathrm{~mW}$ of signal power. Since the intracavity cascaded OPO was configured in a standing wave cavity, the idler output from the CSP OPO could be extracted through both M3 and M4. Owing to the high absorption (>88\%) in the 6-mm-thick fused silica substrate of each mirror in the deep-IR wavelength range, we were only able to measure an idler power of $64 \mu \mathrm{W}$. The extracted idler power can be improved by using suitable ZnSe substrates for M3 and M4. The long-term power stability of the deep-IR idler at a wavelength of $6791 \mathrm{~nm}$ is shown in Fig. 7, confirming a passive power stability better than $1.1 \%$ rms over $2 \mathrm{~h}$. Also shown in the inset of Fig. 7 is the spatial profile of the deep-IR idler beam measured using a pyroelectric camera, indicating a single peak Gaussian distribution.

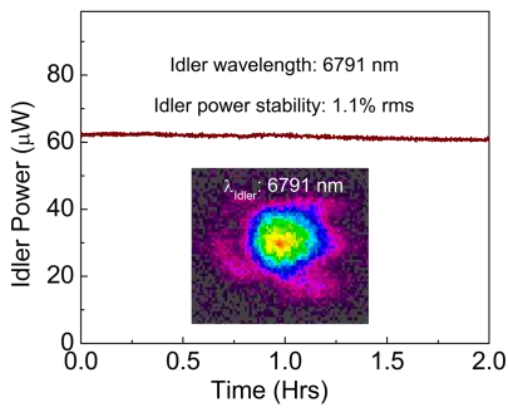

Fig. 7. Long-term power stability of the deep-IR idler from the CSP OPO. Inset: Spatial profile of the deep-IR idler beam.

In conclusion, we have demonstrated a Ti:sapphire-pumped femtosecond OPO for the deep-IR using a novel cascaded intracavity pumping scheme, for the first time to our knowledge. The OPO provides a record idler spectral coverage across 5958-8117 nm using rapid static cavity delay tuning. The deep-IR idler power of $64 \mu \mathrm{W}$ is currently limited by absorption in the fused silica mirror substrates as well as the polarization-dependent losses of the intracavity CSP OPO signal at the prism surfaces. Using ZnSe mirror substrates, AR-coated prisms or chirped mirrors for dispersion compensation, and a KLM Ti:sapphire pump laser of higher power, substantial improvements in the deep-IR output power are expected using the demonstrated technique. The composite cavity design results in excellent long-term passive power stability better than $1.1 \%$ rms over 2 hours, with a FWHM spectral bandwidth as large as large as $658 \mathrm{~nm}$ at $6843 \mathrm{~nm}$. The intracavity cascaded technique is universal and can be deployed in other time-scales and wavelength regions, and using other laser pump sources and nonlinear materials. In particular, the combination of the KLM Ti:sapphire pump laser with technologically mature MgO:PPLN opens up new avenues to exploit the technique for pumping other infrared nonlinear materials such as $\mathrm{AgGaSe}_{2}, \mathrm{ZnGeP}_{2}$ and $\mathrm{OP}-\mathrm{GaAs}$ for deep-IR wavelength generation beyond $\sim 4 \mu \mathrm{m}$.

Funding sources and acknowledgments. We acknowledge support from Spanish MINECO (OPTEX, TEC2012-37853; Severo Ochoa Excellence Grant, SEV-2015-0522), Generalitat de Catalunya (ACCIÓ, VALTEC13-1-0003), EOARD (FA8655-12-1-2128), European Commission (Mid-Tech, H2020-MSCA-ITN-2014), Fundació Privada Cellex, and Generalitat Catalunya (AGAUR, project SGR 2014-2016).

\section{References}

1. S. A. Diddams, L. Hollberg, and V. Mbele, Nature 445, 627 (2007).

2. J. M. Ingram and A. W. Fountain III, Appl. Spectroscopy 61, 1254 (2007).

3. M. Ebrahim-Zadeh, in Solid-State Mid-Infrared Laser Sources, p 184, Springer (2003).

4. P. E. Powers, C. L. Tang, R. J. Ellingson, and W. S. Pelouch, J. Opt. Soc. Am. B 10, 2162 (1993).

5. V. Ramaiah-Badarla, A. Esteban-Martin, and M. Ebrahim-Zadeh, Laser Photonics Rev. 7, L55 (2013).

6. A. Esteban-Martin, O. Kokabee, K. Moutzouris, and M. Ebrahim-Zadeh, Opt. Lett. 34, 428 (2009).

7. F. Adler, K. C. Cossel, M. J. Thorpe, I. Hartl, M. E. Fermann, and J. Ye, Opt. Lett. 34, 1330 (2009).

8. K. T. Zawilski, P. G. Schunemann, T. M. Pollak, D. E. Zelmon, N. C. Fernelius, and F. K. Hopkins, J. Cryst. Growth 312, 1127 (2010).

9. O. Chalus, P. G. Schunemann, K. T. Zawilski, J. Biegert, and M. EbrahimZadeh, Opt. Lett. 35, 4142 (2010).

10. S. Chaitanya Kumar, M. Jelinek, M. Baudisch, K. T. Zawilski, P. G. Schunemann, V. Kubecek, J. Biegert, and M. Ebrahim-Zadeh, Opt. Express 20, 15703 (2012).

11. A. Peremans, D. Lis, F. Cecchet, P. G. Schunemann, K. T. Zawilski, and V. Petrov, Opt. Lett. 34, 3053 (2009).

12. S. Chaitanya Kumar, A. Agnesi, P. Dallocchio, F. Pirzio, G. Reali, K. T. Zawilski, P. G. Schunemann, and M. Ebrahim-Zadeh, Opt. Lett. 36, 3236 (2011)

13. Z. Zhang, D. T. Reid, S. Chaitanya Kumar, M. Ebrahim-Zadeh, P. Schunemann, K. Zawilski, and C. Howle, Opt. Lett. 38, 5110 (2013).

14. S. Chaitanya Kumar, J. Krauth, A. Steinmann, K. T. Zawilski, P. G. Schunemann, H. Giessen, and M. Ebrahim-Zadeh, Opt. Lett. 40, 1398 (2015).

15. S. Chaitanya Kumar, A. Esteban-Martin, T. Ideguchi, M. Yan, S. Holzner, T. W. Hänsch, N. Picqué, and M. Ebrahim-Zadeh, Laser \& Photon. Rev. 8, L86 (2014).

16. S. Marznell, R. Beigang, and R. Wallenstein, Appl. Phys. B 69, 423 (1999). 


\section{References (With titles)}

1. S. A. Diddams, L. Hollberg, and V. Mbele, "Molecular fingerprinting with the resolved modes of a femtosecond laser frequency comb, "Nature 445, 627 (2007).

2. J. M. Ingram and A. W. Fountain III, "Development of a thermal evaporation cell for gas-phase infrared absorption spectroscopy of compounds with low volatility," Appl. Spectroscopy 61, 1254 (2007).

3. M. Ebrahim-Zadeh, "Mid-Infrared ultrafast and continuous-wave optical parametric oscillators," in Solid-State Mid-Infrared Laser Sources, p 184 Springer (2003).

4. P. E. Powers, C. L. Tang, R. J. Ellingson, and W. S. Pelouch, "Recent advances of the Ti:sapphire-pumped high-repetition-rate femtosecond optical parametric oscillator," J. Opt. Soc. Am. B 10, 2162 (1993).

5. V. Ramaiah-Badarla, A. Esteban-Martin, and M. Ebrahim-Zadeh," Self-phase-locked degenerate femtosecond optical parametric oscillator based on $\mathrm{BiB}_{3} \mathrm{O}_{6}$," Laser Photonics Rev. 7, L55 (2013).

6. A. Esteban-Martin, O. Kokabee, K. Moutzouris, and M. Ebrahim-Zadeh, "High-harmonic-repetition-rate, $1 \mathrm{GHz}$ femtosecond optical parametric oscillator pumped by a $76 \mathrm{MHz}$ Ti:sapphire laser," Opt. Lett. 34, 428 (2009).

7. F. Adler, K. C. Cossel, M. J. Thorpe, I. Hartl, M. E. Fermann, and J. Ye, "Phase-stabilized, $1.5 \mathrm{~W}$ frequency comb at 2.8-4.8 $\mu \mathrm{m}$," Opt. Lett. 34, 1330 (2009).

8. K. T. Zawilski, P. G. Schunemann, T. M. Pollak, D. E. Zelmon, N. C. Fernelius, and F. K. Hopkins,"Growth and characterization of large CdSiP 2 single crystals," J. Cryst. Growth 312, 1127 (2010)

9. O. Chalus, P. G. Schunemann, K. T. Zawilski, J. Biegert, and M. EbrahimZadeh, "Optical parametric generation in CdSiP2," Opt. Lett. 35, 4142 (2010).

10. S. Chaitanya Kumar, M. Jelinek, M. Baudisch, K. T. Zawilski, P. G. Schunemann, V. Kubecek, J. Biegert, and M. Ebrahim-Zadeh, "Tunable, high-energy, mid-infrared, picosecond optical parametric generator based on CdSiP,", Opt. Express 20, 15703 (2012).

11. A. Peremans, D. Lis, F. Cecchet, P. G. Schunemann, K. T. Zawilski, and V. Petrov, "Noncritical singly resonant synchronously pumped OPO for generation of picosecond pulses in the mid-infrared near $6.4 \mu \mathrm{m}$, "Opt. Lett. 34, 3053 (2009).

12. S. Chaitanya Kumar, A. Agnesi, P. Dallocchio, F. Pirzio, G. Reali, K. T. Zawilski, P. G. Schunemann, and M. Ebrahim-Zadeh, "Compact, $1.5 \mathrm{~mJ}$, $450 \mathrm{MHz}, \mathrm{CdSiP}_{2}$ picosecond optical parametric oscillator near $6.3 \mu \mathrm{m}$," Opt. Lett. 36, 3236 (2011).

13. Z. Zhang, D. T. Reid, S. Chaitanya Kumar, M. Ebrahim-Zadeh, P. Schunemann, K. Zawilski, and C. Howle, "Femtosecond-laser pumped $\mathrm{CdSiP}_{2}$ optical parametric oscillator producing $100 \mathrm{MHz}$ pulses centered at $6.2 \mu \mathrm{m}$," Opt. Lett. 38, 5110 (2013).

14. S. Chaitanya Kumar, J. Krauth, A. Steinmann, K. T. Zawilski, P. G. Schunemann, H. Giessen, and M. Ebrahim-Zadeh, "High-power femtosecond mid-infrared optical parametric oscillator at $7 \mu \mathrm{m}$ based on CdSiP,", Opt. Lett. 40, 1398 (2015).

15. S. Chaitanya Kumar, A. Esteban-Martin, T. Ideguchi, M. Yan, S. Holzner, T. W. Hänsch, N. Picqué, and M. Ebrahim-Zadeh, "Few-cycle, broadband, mid-infrared optical parametric oscillator pumped by a 20fs Ti:sapphire laser,"Laser \& Photon. Rev. 8, L86 (2014).

S. Marznell, R. Beigang, and R. Wallenstein, "Synchronously pumped femtosecond optical parametric oscillator based on $\mathrm{AgGaSe2}$ tunable from $2 \mu \mathrm{m}$ to $8 \mu \mathrm{m}$," Appl. Phys. B 69, 423 (1999). 